\title{
Как создать цифровое производство в электронной промышленности
}

\author{
В. Ежов
}

\begin{abstract}
Производство электроники сегодня требует внедрения новых подходов, основанных на концепции цифровизации, обеспечивающей сокращение сроков выхода изделий на рынок, повышение качества продукции и снижение затрат. Компания Siemens PLM Software предлагает интегрированную платформу, адаптированную для электронной промышленности, которая охватывает все этапы жизненного цикла изделия: от идеи и разработки опытного образца до запуска серийного производства и эксплуатации. Платформа отражает цифровую стратегию, внедренную компанией на многих предприятиях. 23 мая 2019 года в Москве состоялась конференция, посвященная решениям Siemens PLM Software для технологической подготовки, планирования и управления производством современной электроники.
\end{abstract}

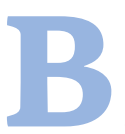

последнее время производители электроники сталкиваются со значительными рыночными вызовами. Требования к качеству и функционалу изделия постоянно растут, повышается спрос на устройства, подключенные к Интернету. Пользователи хотят получать новые модели быстрее, причем все чаще им требуются уникальные варианты исполнения продукта. Стараясь соответствовать требованиям рынка, компании-производители прилагают усилия, чтобы ускорить внедрение новых технологий (3D-печать, робототехнические системы и т.д.), однако в результате возрастает сложность изделий и процессов их изготовления. Необходимость сокращения сроков вывода продукта на рынок сводит к минимуму период отработки новой технологии. Кастомизация изделий сдерживается негибкостью существующих производственных систем.

Ответом на эти трудности стало внедрение цифровых технологий на различных этапах процесса подготовки и управления производством. Цифровизация повышает качество технологического проектирования, в результате чего возрастает эффективность всех производственных операций. Однако проведенное компанией Gartner исследование показало, что пока цифровизация не дает ожидаемого эффекта. По итогам опроса представителей приборостроительных предприятий США выяснилось, что 80\% предприятий реализуют проекты по цифровизации, но при этом менее половины отметили, что инвестиции в цифровые технологии не приводят к росту прибыли. Проанализировав полученные результаты, компания Gartner пришла к интересному выводу - внедрение новых технологий неэффективно, если на предприятии нет современной гибкой производственной системы, которая быстро реагирует на рыночные изменения, позволяет сопровождать множество разнообразных производственных заказов параллельно, предоставляет необходимую и корректную информацию для других подразделений предприятия: отдела логистики, финансов, менеджмента и т. п. Иными словами, для успешной цифровизации модели ведения бизнеса оказываются важнее прогресса в цифровых технологиях.

Siemens PLM Software предлагает раскрыть потенциал цифровизации на основе новой модели функционирования предприятия, объединяющей процессы проектирования, производства и обслуживания изделия. Siemens уделяет большое внимание созданию сквозных систем, охватывающих все этапы жизненного цикла изделия. За разработку таких систем в концерне отвечает подразделение - Siemens PLM Software.

В своем выступлении на конференции вице-президент, генеральный менеджер Siemens PLM Software в России и СНГ Виктор Беспалов отметил, что сегодня Siemens PLM Software - единственная в мире компания, которая обладает полным набором PLM-решений как для машиностроительных предприятий, так и для компаний в сфере электроники и приборостроения. За последние два года концерн приобрел ряд крупных компаний, деятельность которых связана с приборостроением. В первую очередь стоит выделить Mentor Graphics - ведущего мирового поставщика электронных CAD. Сего приобретением Siemens получил недостающее звено в цепочке жизненного цикла электронного продукта.

Известно, что Siemens - один из мировых лидеров идеологии цифрового предприятия и «Индустрии 4.0». По словам Виктора Беспалова, компания не просто разработчик цифровой стратегии производства, но и крупнейший 


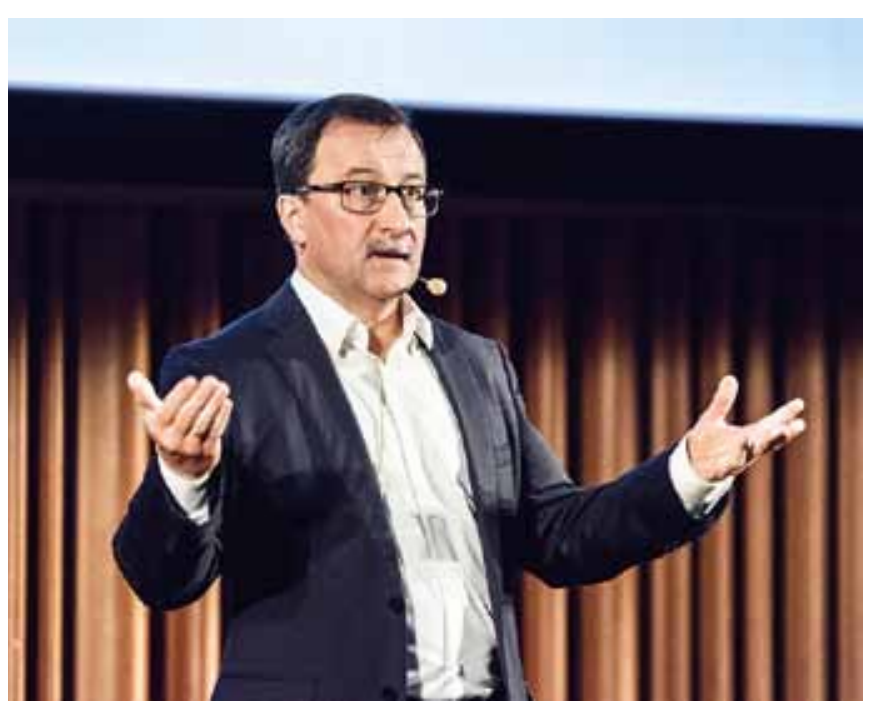

Виктор Беспалов, вице-президент, генеральный менеджер Siemens PLM Software в России и СНГ

потребитель этих технологий, которые успешно используются концерном на своих предприятиях.

Основу подхода к цифровизации от Siemens PLM Software составляет концепция цифровых двойников. Это целостный подход, который объединяет все технологии цифровизации в единую модель. Цифровой двойник - это точная виртуальная модель изделия либо технологического процесса. Цифровой двойник продукта полный набор цифровых данных, которые описывают не только геометрию, механику изделия, но и электронные компоненты, физические процессы, управляющее программное обеспечение. При запуске нового продукта в производство нужно выполнить технологическую подготовку производства, инжиниринг, а в дальнейшем обеспечивать управление реальным производством. То есть необходим также цифровой двойник производства. Эти модели применяются для анализа реальных процессов еще до начала изготовления изделия или проектирования технологических операций с целью их оптимизации. Следует учитывать, что поступающая в режиме реального времени информация постоянно обновляет цифровые двойники, гарантируя их максимальную точность. Передача сведений об эксплуатации изделия на этап проектирования и испытания будущих изделий обеспечивает их дальнейшее совершенствование. В аналогичном порядке информация о выполнении реальных технологических операций помогает повышать эффективность технологических процессов

Виктор Беспалов отметил, что за последние десять лет концерн Siemens вложил в развитие технологий, связанных с цифровизацией, более 10 млрд евро. Среди компаний, приобретенных Siemens для развития приборостроительного направления, наряду с Mentor Graphics интерес представляет Mendix. Программные решения этой компании позволяют быстро создавать приложения и интеграционные решения на основе систем, которые использует заказчик, что важно в условиях быстро меняющихся требований рынка.

Руководитель бизнес-направления «Приборостроение и Роскосмос" Siemens PLM Software Дмитрий Собянин, выступая на конференции, отметил, что компания предлагает полный комплекс решений по всем направлениям создания нового изделия. Наряду с CAE- и CAD-системами в портфолио компании входят PLM- и MES-системы, причем обеспечивается их интеграция с широко распространенными ЕRP-системами, в частности от компаний SAP или 1C.

Проектирование изделия на платформе Siemens PLM Software ведется по четырем координатам: корпус (механическая часть), электроника (от Ис до печатных плат), коммутационная часть (жгуты) и программное обеспечение. За электронику отвечает семейство решений Mentor Graphics, которые обеспечивают проектирование сложных печатных плат, СБИС, СнК, СвК. Корпуса и другие сложные конструкции, робототехнические системы, мехатронные устройства проектируются с помощью семейства NX. Задачи, связанные с разработкой кабельно-жгутовых соединений, решает Mentor Graphics Capital. В процессе разработки изделия производится верификация технических характеристик изделия, предусмотренных Т3,

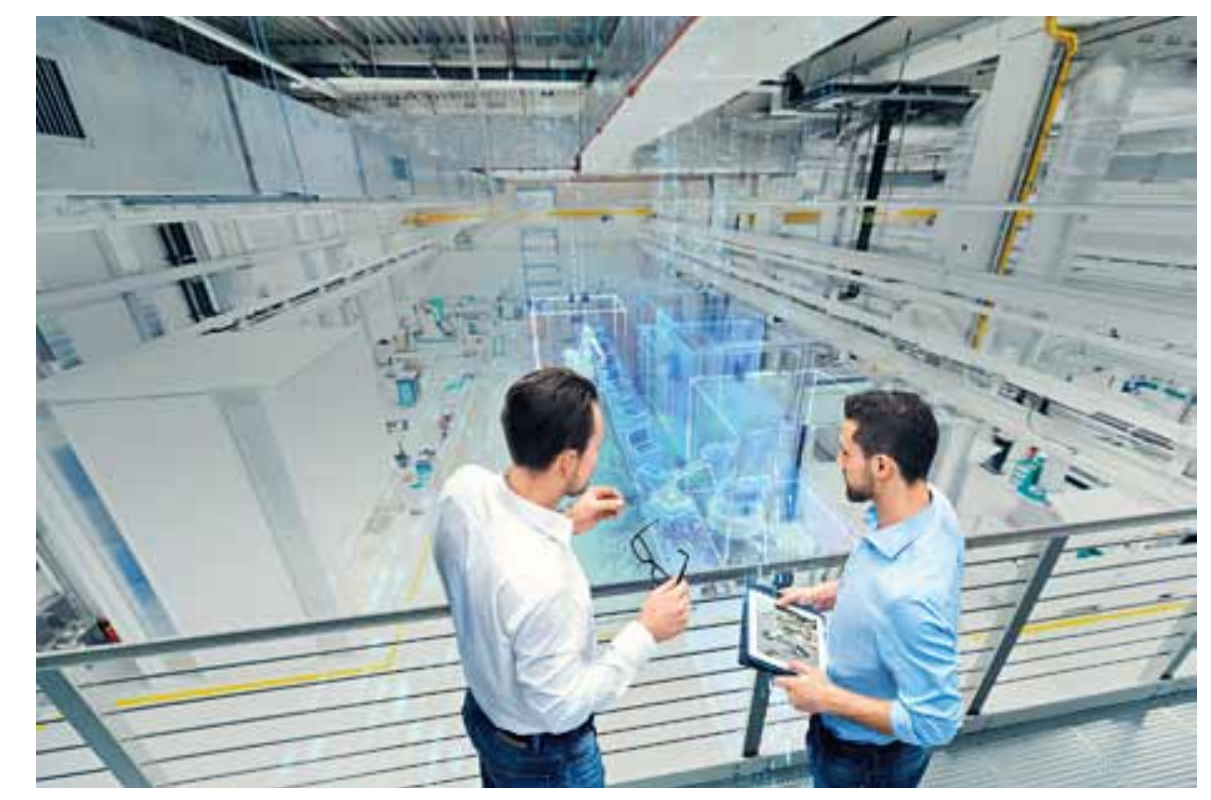




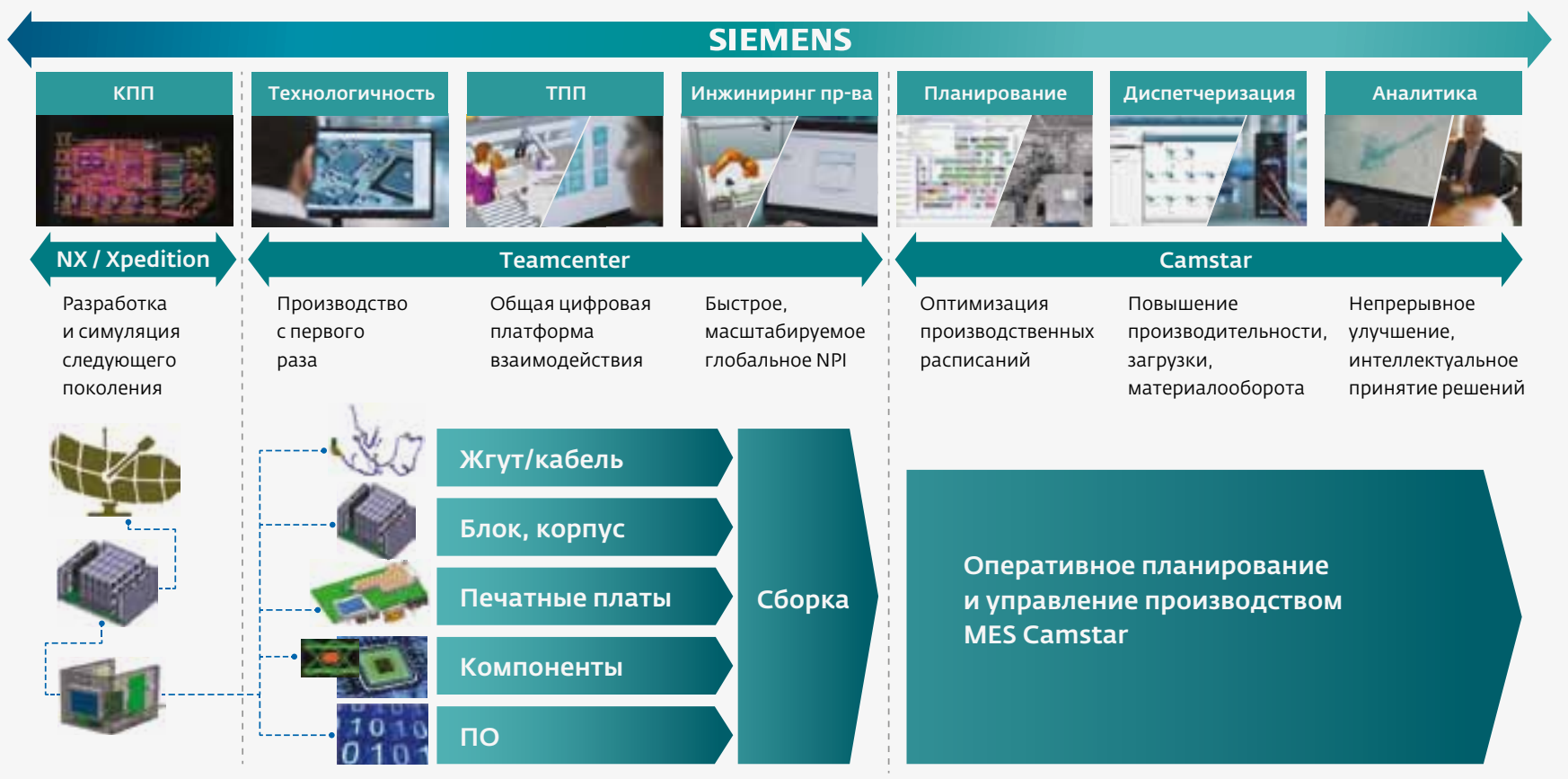

в том числе для электроники - функциональный анализ ИС, посттопологический анализ печатных плат, анализ целостности сигналов и питания и т.д. Для прибора в целом выполняется комплекс инженерных расчетов, связанных с прочностными характеристиками, виброустойчивостью, тепловыми режимами. Кроме того, запускается процесс верификации расчетных моделей по данным испытаний. Автоматизирован весь маршрут создания ПО от разработки требований и написания кода до тестирования и управления версиями.

Параллельно с проектированием изделия средствами Siemens PLM Software ведется разработка технологии изготовления - печатных плат, механической конструкции, кабельных жгутов, включая разработку технологической документации по ЕСТД. Применяются различные системы DFM-анализа (такие как Valor NPI), которые обеспечивают технологичность разрабатываемого изделия, сокращая тем самым цикл согласования того или иного решения между конструкторами и технологами. Параллельно разрабатываются управляющие программы для всех типов технологического оборудования: станков с чПу, оборудования печатного монтажа, тестовых систем и т.д.

После завершения разработки изделия и технологических процессов начинается этап его производства. Инструменты Siemens PLM Software позволяют решать задачи по оперативному управлению производством (печатных плат, готовых изделий), оперативно-календарному планированию, управлению качеством и др.
Все эти компоненты платформы работают в рамках единой информационной среды, все задачи решаются на основе цифрового двойника изделия, благодаря чему сокращаются сроки разработки, внесения изменений и запуска производства. Управление данными об изделии на каждом этапе его жизненного цикла производится с помощью PLM-платформы Teamcenter, a MES-система Camstar Electronics управляет всеми процессами электронного производства.

Дмитрий Собянин подчеркнул, что внедрение решений Siemens PLM Software на предприятии не означает замену всех уже используемых систем. Специалисты компании могут встроить работающие на предприятии системы в единое PLM-решение. у Siemens накоплен колоссальный опыт работы в области автоматизации процессов в электронной промышленности и приборостроении. Технологии Siemens позволяют выпускать изделия по конкурентным ценам, обеспечивают возможность принятия управленческих решений на основе корректных цифровых данных, полученных на этапе производства и эксплуатации изделия. Цифровые технологии действительно работают. Примером служит завод Siemens по производству промышленных контроллеров в г. Амберг (Германия). Внедрение технологий «Индустрии 4.0» позволило достичь впечатляющих результатов - предприятие выпускает 12 млн ПЛК в год или одно изделие в секунду, время от получения заказа до отгрузки готового изделия составляет всего 24 ч, а качество изделий составляет не более 10 дефектов на миллион. 


\section{HAHECEHИE ГААЬВАНИЧЕСКИХ ПОКРЫТИЙ}

из никеля (Ni) и золота (Au)

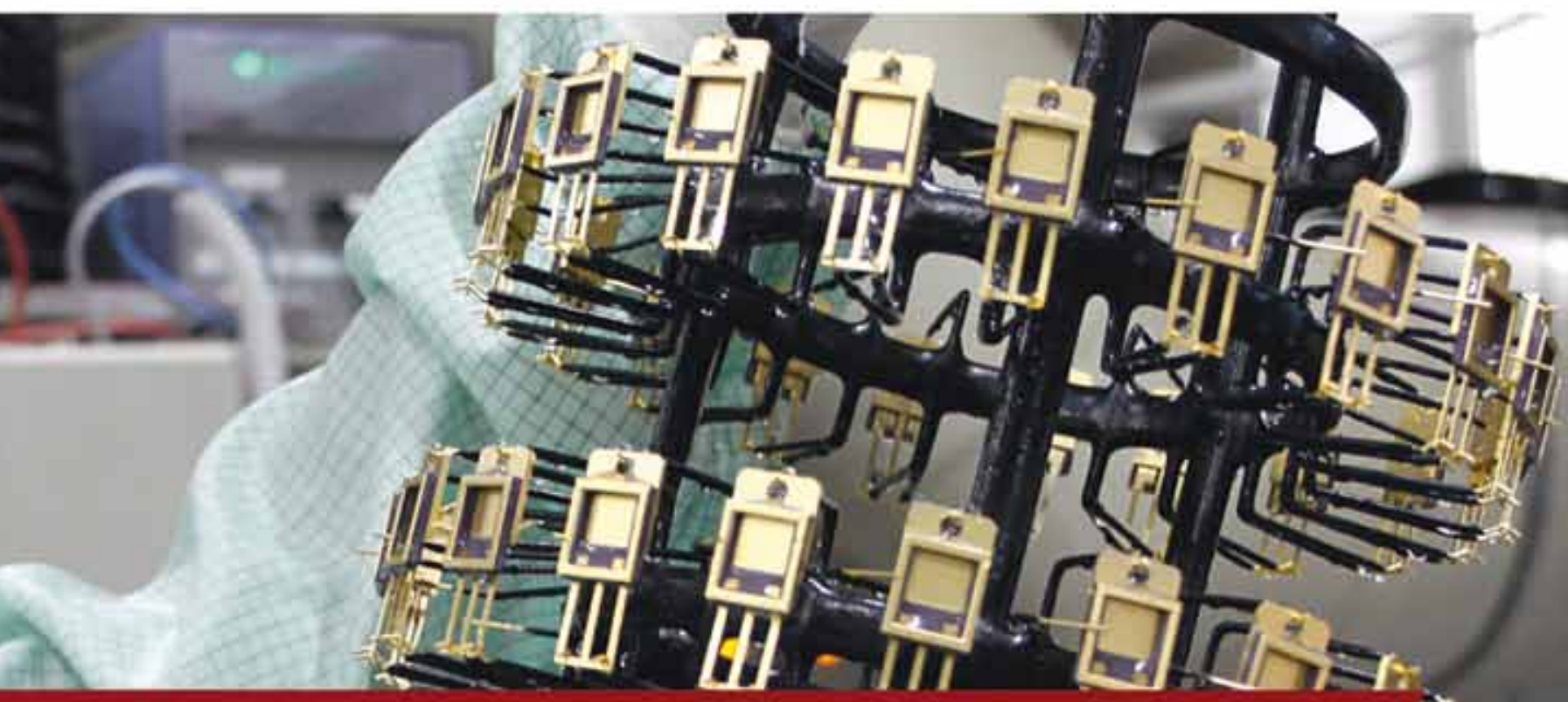

Самый современный технологический прочесс и многоступенчатый контро^ь качества гарантируют выполнение самых сложных запросов

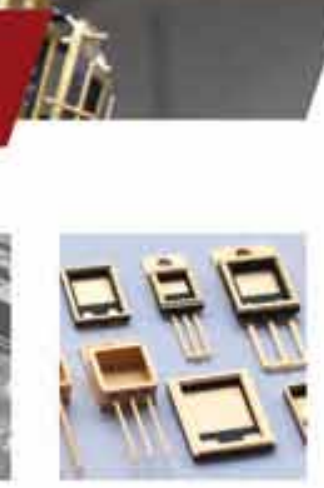

НА ПРОИЗВОДСТВЕ КОМПАНИИ «ТЕСТПРИБОР» ОСВОЕНЫ СЛЕДУЮЩИЕ ТЕХНОЛОГИИ НАНЕСЕНИЯ МЕТАЛЛИЧЕСКИХ ПОКРЫТИЙ:

- электрохимическое никелирование из электролитов различных составов

- электрохимическое никелирование сплавом никель-фосфор
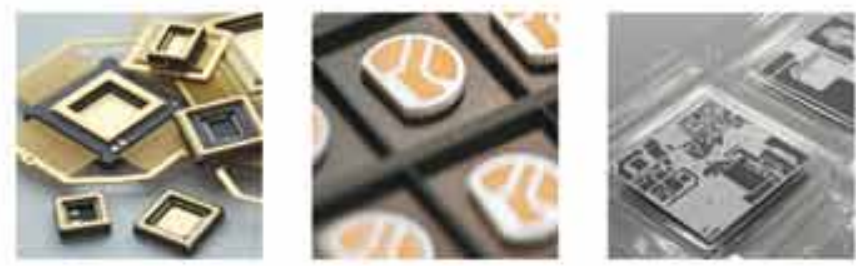